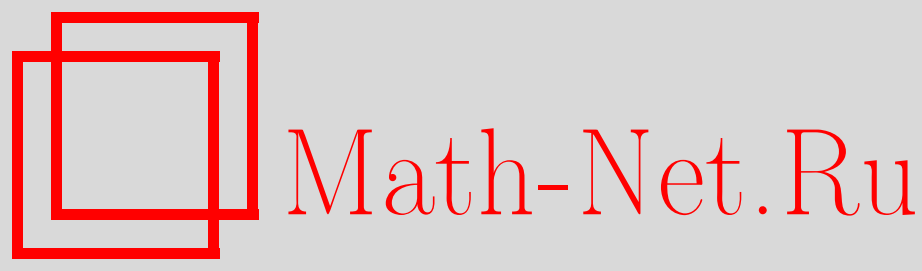

А. А. Славнов, Теория Янга-Миллса как безмассовый предел массивной калибровочно-инвариантной модели, TMФ, 2013, том 175, номер 1, 3-10

DOI: https://doi.org/10.4213/tmf8451

Использование Общероссийского математического портала Math-Net.Ru подразумевает, что вы прочитали и согласны с пользовательским соглашением http://www.mathnet.ru/rus/agreement

Параметры загрузки:

IP: 35.174 .16 .151

26 апреля 2023 г., 17:21:20

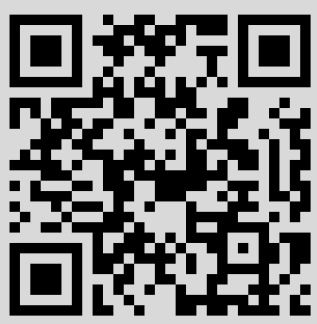




\section{ТЕОРИЯ ЯНГА-МИЛЛСА}

\section{КАК БЕЗМАССОВЫЙ ПРЕДЕЛ МАССИВНОЙ КАЛИБРОВОЧНО-ИНВАРИАНТНОЙ МОДЕЛИ}

Построена калибровочно-инвариантная инфракрасная регуляризация теории Янга-Миллса, применимая вне рамок теории возмущений.

Ключевые слова: поле Янга-Миллса, инфракрасные расходимости, регуляризация.

DOI: $10.4213 / \operatorname{tmf} 8451$

\section{1. ВВЕДЕНИЕ}

Хорошо известно, что теория Янга-Миллса не может быть получена как предел при $m \rightarrow 0$ массивной теории Янга-Миллса. Продольная поляризация массивного векторного поля не отщепляется в пределе нулевой массы и дает ненулевой вклад [1]. Недавно было показано [2], что массивная неабелева калибровочно-инвариантная модель, включающая дополнительные возбуждения, может быть использована как калибровочно-инвариантная инфракрасная регуляризация теории Янга-Миллса. Однако процедуру, предложенную в работе [2], можно применить только в рамках теории возмущений по константе связи. С другой стороны, в присутствии инфракрасных сингулярностей теория возмущений неприменима, хотя регуляризация, предложенная в работе [2], может быть полезна для вычисления функций Грина калибровочно-инвариантных операторов.

В настоящей работе мы предлагаем калибровочно-инвариантную неабелеву модель, которая может быть использована в качестве инфракрасной регуляризации теории Янга-Миллса как в теории возмущений, так и вне ее.

\section{2. МАССИВНАЯ НЕАБЕЛЕВА}

\section{КАЛИБРОВОЧНО-ИНВАРИАНТНАЯ МОДЕЛЬ}

В работах [3]-[5] указывалось, что невозможность однозначного выбора калибровочного условия вне рамок теории возмущений не является внутренним свойством теории Янга-Миллса, а связана с ее конкретной формулировкой. Добавляя новые

* Математический институт им. В. А. Стеклова РАН, Москва, Россия. E-mail: slavnov@mi.ras.ru

${ }^{\dagger}$ Московский государственный университет им. М. В. Ломоносова, Москва, Россия 
возбуждения, которые асимптотически отщепляются, можно проквантовать неабелеву калибровочную модель, не нарушая релятивистской инвариантности как в теории возмущений, так и вне ее.

Имея это ввиду, мы предлагаем использовать для инфракрасной инвариантной регуляризации теории Янга-Миллса следующий лагранжиан:

$$
\begin{aligned}
L= & -\frac{1}{4} F_{\mu \nu}^{a} F_{\mu \nu}^{a}-m^{-2}\left(D^{2} \tilde{\phi}\right)^{*}\left(D^{2} \tilde{\phi}\right)+\left(D_{\mu} e\right)^{*}\left(D_{\mu} b\right)+\left(D_{\mu} b\right)^{*}\left(D_{\mu} e\right)+ \\
& +\alpha^{2}\left(D_{\mu} \tilde{\phi}\right)^{*}\left(D_{\mu} \tilde{\phi}\right)-\alpha^{2} m^{2}\left(b^{*} e+e^{*} b\right),
\end{aligned}
$$

где $\phi$ - двухкомпонентный комплексный дублет и

$$
\tilde{\phi}=\phi-\hat{\mu}, \quad \hat{\mu}=\left(0, \mu \sqrt{2} g^{-1}\right) .
$$

Здесь $\mu$ - произвольная константа, имеющая размерность массы. В формуле $(1) D_{\mu}$ обозначает обычную ковариантную производную. Для экономии места мы рассматриваем группу $S U(2)$.

В дальнейшем мы будем пользоваться параметризацией $\phi$ в терминах эрмитовых компонент:

$$
\phi=\left(\frac{i \phi^{1}+\phi^{2}}{\sqrt{2}}\left(1+\frac{g}{2 \mu} \phi^{0}\right), \frac{\phi^{0}-i \phi^{3}\left(1+g /(2 \mu) \phi^{0}\right)}{\sqrt{2}}\right) .
$$

Комплексные антикоммутирующие скалярные поля $b, e$ будут параметризованы следующим образом:

$$
b=\left(\frac{i b^{1}+b^{2}}{\sqrt{2}}, \frac{b^{0}-i b^{3}}{\sqrt{2}}\right)\left(1+\frac{g}{2 \mu} \phi^{0}\right), \quad e=\left(\frac{i e^{1}+e^{2}}{\sqrt{2}}, \frac{e^{0}-i e^{3}}{\sqrt{2}}\right),
$$

где компоненты $e^{\alpha}$ эрмитовы, а компоненты $b^{\alpha}$ антиэрмитовы. Мы пользуемся этой специальной параметризацией классических полей, поскольку хотим полностью избавиться от неоднозначности при выборе калибровки для квантования.

Лагранжиан (1), очевидно, инвариантен относительно “сдвинутых" калибровочных преобразований

$$
\begin{aligned}
A_{\mu}^{a} & \rightarrow A_{\mu}^{a}+\partial_{\mu} \eta^{a}-g \epsilon^{a b c} A_{\mu}^{b} \eta^{c}, \\
\phi^{a} & \rightarrow \phi^{a}+\frac{g}{2} \epsilon^{a b c} \phi^{b} \eta^{c}+\eta^{a} \mu+\frac{g^{2}}{4 \mu} \phi^{a} \phi^{b} \eta^{b}, \\
\phi^{0} & \rightarrow \phi^{0}-\frac{g}{2}\left(\phi^{a} \eta^{a}\right)\left(1+\frac{g}{2 \mu} \phi^{0}\right), \\
b^{a} & \rightarrow b^{a}+\frac{g}{2} \epsilon^{a b c} b^{b} \eta^{c}+\frac{g}{2} b^{0} \eta^{a}+\frac{g^{2}}{4 \mu} b^{a} \phi^{b} \eta^{b}, \\
e^{a} & \rightarrow e^{a}+\frac{g}{2} \epsilon^{a b c} e^{b} \eta^{c}+\frac{g}{2} e^{0} \eta^{a}, \\
b^{0} & \rightarrow b^{0}-\frac{g}{2} b^{a} \eta^{a}+\frac{g^{2}}{4 \mu}\left(\phi^{a} \eta^{a}\right) b^{0}, \\
e^{0} & \rightarrow e^{0}-\frac{g}{2} e^{a} \eta^{a} .
\end{aligned}
$$


Этот лагранжиан также инвариантен относительно преобразований суперсимметрии

$$
\phi \rightarrow \phi-b \epsilon, \quad e \rightarrow e-\frac{D^{2}(\phi-\hat{\mu})}{m^{2}} \epsilon, \quad b \rightarrow b,
$$

где $\epsilon$ - постоянный антикоммутирующий параметр. Эта симметрия играет важную роль в доказательстве отщепления нефизических возбуждений. Лагранжиан (1) инвариантен относительно преобразований (6) при любом $\alpha$, но при $\alpha=0$ эти преобразования являются также нильпотентными. Заметим, что для дальнейшего обсуждения нам понадобятся только существование сохраняющегося заряда $Q$ и нильпотентность асимптотического заряда $Q_{0}$, поскольку физический спектр определяется асимптотической динамикой (мы пользуемся стандартной задачей рассеяния, полагая, что асимптотический спектр совпадает со спектром свободного гамильтониана). В рассматриваемом случае нильпотентность асимптотического заряда требует обращения $\alpha$ в нуль. Массивная теория с $\alpha \neq 0$ калибровочно-инвариантна, но не унитарна. Это может показаться странным, поскольку обычно калибровочная инвариантность является достаточным условием унитарности, так как в этом случае можно легко перейти от перенормируемой калибровки к унитарной, в которой спектр включает только физические возбуждения. В данном случае "унитарной" калибровки не существует. Даже в калибровке $\phi^{a}=0$ присутствуют нефизические возбуждения.

Для калибровочных преобразований (5) калибровка $\phi^{a}=0$ является однозначной как в теории возмущений, так и вне ее. Действительно, если $\phi^{a}=0$, то при калибровочных преобразованиях (5) вариация переменных $\phi^{a}$ равна $\delta \phi^{a}=\mu \eta^{a}$, и условие $\phi^{a}=0$ приводит к тому, что $\eta^{a}=0$. В этой калибровке переменные $\phi^{a}$ параметризуются как обычно:

$$
\phi=\left(0, \frac{\phi^{0}}{\sqrt{2}}\right)+\hat{\mu} .
$$

Очевидно, что при $\alpha \neq 0$ лагранжиан (1) описывает массивное векторное поле и не порождает инфракрасных сингулярностей. В терминах "сдвинутых" полей лагранжиан (1) принимает вид

$$
\begin{aligned}
L= & -\frac{1}{4} F_{\mu \nu}^{a} F_{\mu \nu}^{a}-m^{-2}\left(D^{2} \phi\right)^{*}\left(D^{2} \phi\right)+m^{-2}\left(D^{2} \phi\right)^{*}\left(D^{2} \hat{\mu}\right)+ \\
& +m^{-2}\left(D^{2} \hat{\mu}\right)^{*}\left(D^{2} \phi\right)-m^{-2}\left(D^{2} \hat{\mu}\right)^{*}\left(D^{2} \hat{\mu}\right)+\left(D_{\mu} e\right)^{*}\left(D_{\mu} b\right)+ \\
& +\left(D_{\mu} b\right)^{*}\left(D_{\mu} e\right)+\alpha^{2}\left(D_{\mu} \phi\right)^{*}\left(D_{\mu} \phi\right)-\alpha^{2}\left(D_{\mu} \phi\right)^{*}\left(D_{\mu} \hat{\mu}\right)- \\
& -\alpha^{2}\left(D_{\mu} \hat{\mu}\right)^{*}\left(D_{\mu} \phi\right)+\alpha^{2}\left(D_{\mu} \hat{\mu}\right)^{*}\left(D_{\mu} \hat{\mu}\right)-\alpha^{2} m^{2}\left(b^{*} e+e^{*} b\right),
\end{aligned}
$$

и член

$$
\alpha^{2}\left(D_{\mu} \hat{\mu}\right)^{*}\left(D_{\mu} \hat{\mu}\right)=\frac{\alpha^{2} \mu^{2}}{2} A_{\mu}^{2}
$$

порождает массу векторного поля.

Член

$$
\frac{1}{m^{2}}\left(D^{2} \hat{\mu}\right)^{*}\left(D^{2} \hat{\mu}\right)=\frac{\mu^{2}}{2 m^{2}}\left[\left(\partial_{\mu} A_{\mu}\right)^{2}+\frac{g^{2}}{2}\left(A^{2}\right)^{2}\right]
$$


делает теорию перенормируемой при любом $\alpha$. Чтобы избежать осложнений, связанных с присутствием в спектре теории Янга-Миллса при $\alpha=0$ духовых полюсов, мы положим $\mu^{2}=m^{2}$.

Эффективный лагранжиан в калибровке $\phi^{a}=0$ может быть записан в виде

$$
L_{\mathrm{ef}}=L+\lambda^{a} \phi^{a}-\mu \bar{c}^{a} c^{a},
$$

где $L$ - лагранжиан (8), а $\bar{c}^{a}, c^{a}$ - нединамические духовые поля.

Инвариантность лагранжиана (8) относительно калибровочных преобразований (5) и преобразований суперсимметрии (6) приводит к инвариантности эффективного лагранжиана относительно одновре́менных БРСТ-преобразований, отвечающих преобразованиям (5) и преобразованиям суперсимметрии (6). Если $s_{1}$ - нильпотентный оператор, соответствующий одновременному применению преобразований суперсимметрии и БРСТ-преобразований, а преобразования полей имеют вид

$$
s_{1} \lambda^{a}=0, \quad s_{1} c^{a}=-\frac{g}{2} \epsilon_{a b c} c^{b} c^{c}, \quad s_{1} \bar{c}^{a}=\lambda^{a},
$$

то эффективный лагранжиан может быть также записан в форме

$$
L_{\mathrm{ef}}=L+s_{1} \bar{c}^{a} \phi^{a}=L(x)+\lambda^{a} \phi^{a}-\bar{c}^{a}\left(\mu c^{a}-b^{a}\right) .
$$

Как было указано в работе [5], в континуальном интеграле, который определяет вакуумное ожидание любого оператора, соответствующего какой-либо наблюдаемой, можно выполнить интегрирование по полям $\lambda, \bar{c}^{a}, c^{a}$. Это приведет к замене $c^{a}=b^{a} \mu^{-1}$, и в результате такого интегрирования мы получим эффективный лагранжиан, инвариантный относительно преобразований, которые являются суммой БРСТ-преобразования и преобразований суперсиметрии (6), причем в этих преобразованиях $c^{a}$ заменено на $b^{a} \mu^{-1}$ :

$$
\begin{array}{rlrl}
\delta A_{\mu}^{a}=D_{\mu} b^{a} \mu^{-1} \epsilon, & \\
\delta \phi^{a} & =0, & \delta \phi^{0} & =-b^{0}\left(1+\frac{g}{2 \mu} \phi^{0}\right) \epsilon, \\
\delta e^{a} & =\left(\frac{g}{2 \mu} \epsilon^{a b c} e^{b} b^{c}+\frac{g e^{0} b^{a}}{2 \mu}+i \frac{D^{2}(\tilde{\phi})^{a}}{\mu^{2}}\right) \epsilon, & \delta e^{0} & =\left(-\frac{g e^{a} b^{a}}{2 \mu}-\frac{D^{2}(\tilde{\phi})^{0}}{\mu^{2}}\right) \epsilon, \\
\delta b^{a} & =\frac{g}{2 \mu} \epsilon^{a b c} b^{b} b^{c}, & \delta b^{0} & =0 .
\end{array}
$$

Поскольку преобразования (14) сохраняют условие $\phi^{a}=0$, мы опустили в этих преобразованиях все члены, пропорциональные $\phi^{a}$. Для асимптотического гамильтониана эти преобразования принимают вид

$$
\begin{array}{rlrl}
\delta A_{\mu}^{a}=\partial_{\mu} b^{a} \mu^{-1} \epsilon, & \\
\delta \phi^{a} & =0, & \delta \phi^{0} & =-b^{0} \epsilon, \\
\delta e^{a} & =\partial_{\mu} A_{\mu}^{a} \mu^{-1}, & \delta e^{0} & =-\partial^{2} \phi^{0} \mu^{-2}, \\
\delta b^{a} & =0, & \delta b^{0} & =0 .
\end{array}
$$


Согласно теореме Нётер инвариантность относительно преобразований (14) порождает сохраняющийся заряд $Q$, и можно выбрать в качестве физических асимптотических состояний состояния, удовлетворяющие условию

$$
\widehat{Q}_{0}|\psi\rangle_{\text {as }}=0,
$$

где

$$
\begin{aligned}
Q_{0}= & \int d^{3} x\left[\left(\partial_{0} A_{i}^{a}-\partial_{i} A_{0}^{a}\right) \mu^{-1} \partial_{i} b^{a}-\mu^{-1} \partial_{\nu} A_{\nu}^{a} \partial_{0} b^{a}+\right. \\
& \left.+\mu^{-2} \partial^{2}\left(\partial_{0} \phi^{0}\right) b^{0}-\mu^{-2} \partial_{0} b^{0} \partial^{2} \phi^{0}-\mu \alpha^{2} b^{a} A_{0}^{a}\right] .
\end{aligned}
$$

В силу сохранения нётеровского заряда это условие инвариантно относительно динамики.

Пользуясь каноническими переменными Остроградского для систем, описываемых лагранжианами с высшими производными, можно переписать равенство (17) в виде

$$
Q_{0}=\int d^{3} x\left[-\left(\partial_{i} p_{i}^{a} b^{a} \mu^{-1}+\mu \alpha^{2} b^{a} A_{0}^{a}\right)+\mu^{-1} p_{0}^{a} \partial_{0} b-\left(p_{1}-\alpha^{2} \varphi_{2}\right) b+p_{2} \partial_{0} b\right] .
$$

Здесь

$$
\begin{gathered}
p_{i}^{\alpha}=\frac{\partial L}{\partial \dot{A}_{i}^{\alpha}}, \quad p_{i}=\frac{\delta L}{\delta \dot{\phi}_{i}}-\frac{d}{d t} \frac{\delta L}{\delta \ddot{\phi}_{i}}, \\
H=p_{i}^{\alpha} \dot{A}_{i}^{\alpha}+p_{b} \dot{b}+p_{e} \dot{e}+p_{1} \phi_{2}+p_{2} \partial_{0} \phi_{2}-L, \\
p_{i}^{a}=\dot{A}_{i}^{a}-\partial_{i} A_{0}^{a}, \quad p_{0}^{a}=-\partial_{\nu} A_{\nu}^{a}, \quad p_{b}^{a}=\dot{e}^{a}, \quad p_{e}^{a}=\dot{b}^{a}, \\
\varphi_{1}=\phi^{0}, \quad \varphi_{2}=\dot{\phi}^{0}, \quad p_{1}=\mu^{-2} \partial^{2} \dot{\phi}^{0}+\alpha^{2} \dot{\phi}^{0}, \quad p_{2}=-\mu^{-2} \partial^{2} \phi^{0}, \\
b^{0}=b, \quad e^{0}=e, \quad p_{b}=\dot{e}, \quad p_{e}=\dot{b} .
\end{gathered}
$$

В этих обозначениях асимптотический гамильтониан выглядит следующим образом:

$$
\begin{aligned}
H_{0}= & \frac{p_{i}^{2}}{2}-\frac{p_{0}^{2}}{2}-\partial_{i} p_{i}^{a} A_{0}^{a}+p_{0}^{a} \partial_{i} A_{i}^{a}+\frac{1}{4}\left(F_{i j}^{a}\right)^{2}+p_{b}^{a} p_{e}^{a}+\partial_{i} b^{a} \partial_{i} e^{a}+ \\
& +p_{b} p_{e}+\partial_{i} b \partial_{i} e+p_{1} \varphi_{2}-\frac{\mu^{2}}{2} p_{2}^{2}+p_{2} \Delta \varphi_{1}- \\
& -\frac{\alpha^{2} \mu^{2}}{2} A_{0}^{2}+\frac{\alpha^{2} \mu^{2}}{2} A_{i}^{2}+\alpha^{2} \mu^{2} b e-\frac{\alpha^{2}}{2} \varphi_{2}^{2}+\frac{\alpha^{2}}{2} \partial_{i} \varphi_{1} \partial_{i} \varphi_{1} .
\end{aligned}
$$

Мы хотим доказать, что лагранжиан (4) действительно описывает инфракрасную регуляризацию теории Янга-Миллса. Это значит, что при $\alpha \neq 0$ он отвечает массивной калибровочно-инвариантной теории, а в пределе $\alpha=0$ описывает обычные трехмерно-поперечные поляризации поля Янга-Миллса. Разумеется, при $\alpha \neq 0$ спектр соответствующего гамильтониана включает также нефизические возбуждения.

В пределе $\alpha=0$ выживают только две первые строки в формуле (20). Они содержат члены, зависящие только от полей $A_{0}, A_{i}$ и соответствующих канонических моментов, которые совпадают с обычным свободным гамильтонианом поля 
Янга-Миллса в диагональной фейнмановской калибровке, и от полей $\phi^{0}, b^{0}, e^{0}$. Поля $b^{a}, e^{a}$ играют роль духов Фаддеева-Попова. Обычные аргументы показывают, что продольная и временна́я компоненты поля Янга-Миллса и поля́ $b^{a}, e^{a}$ отщепляются от остальных компонент, и физические состояния могут содержать только поперечные компоненты поля Янга-Миллса и переменные, соответствующие полям $\phi^{0}, b^{0}, e^{0}$. Ниже мы покажем, что поля $\phi^{0}, b^{0}, e^{0}$ также отщепляются.

Из равенства (20) следует, что асимптотические импульсы $p_{1}, p_{2}$ удовлетворяют уравнениям Клейна-Гордона $\partial^{2} p_{1,2}=0$. Следовательно, эти импульсы допускают стандартное разложение

$$
p_{1,2}(x)=(2 \pi)^{-3 / 2} \int d^{3} k \frac{i \sqrt{\omega}}{2}\left(a_{p_{1,2}}^{+}(\mathbf{k}) \mathrm{e}^{i k x}-a_{p_{1,2}}^{-}(\mathbf{k}) \mathrm{e}^{-i k x}\right),
$$

где $k_{0}=\omega=\sqrt{\mathbf{k}^{2}}$. Аналогичное разложение можно написать для асимптотических полей $b, \dot{b}$ в форме (и для $e, \dot{e}$ в точно такой же форме при замене $b, \dot{b} \rightarrow e, \dot{e})$ :

$$
\begin{aligned}
& b(x)=(2 \pi)^{-3 / 2} \int d^{3} k \frac{1}{\sqrt{2 \omega}}\left(b^{+}(\mathbf{k}) \mathrm{e}^{i k x}+b^{-}(\mathbf{k}) \mathrm{e}^{-i k x}\right), \\
& \dot{b}(x)=(2 \pi)^{-3 / 2} \int d^{3} k \frac{i \sqrt{\omega}}{\sqrt{2}}\left(b^{+}(\mathbf{k}) \mathrm{e}^{i k x}-b^{-}(\mathbf{k}) \mathrm{e}^{-i k x}\right),
\end{aligned}
$$

где $k_{0}=\omega=\sqrt{\mathbf{k}^{2}}$. Канонические переменные, описывающие поля $\phi^{0}$, вообще говоря, не являются осцилляторными и не могут быть представлены операторами в обычном фоковском пространстве.

Асимптотические поля $\varphi_{1,2}, p_{1,2}$ удовлетворяют уравнениям движения, которые вытекают из гамильтониана (20):

$$
\dot{\phi}_{1}=\varphi_{2}, \quad \dot{\phi}_{2}+\mu^{2} p_{2}-\Delta \varphi_{1}=0, \quad \dot{p}=\Delta p_{2}, \quad \dot{p}=p_{1}
$$

В соответствии с этими уравнениями часть асимптотического БРСТ-заряда

$$
\widetilde{Q}_{0}=\int d^{3} x\left(p_{2} \dot{b}+p_{1} b\right)
$$

зависящая от переменных $p_{1,2}$, сохраняется и, следовательно, не зависит от времени. Поэтому мы можем положить $x_{0}$ в переменных $p_{1,2}, b, \dot{b}$ равным нулю.

Пользуясь уравнениями $(21),(22)$, получим

$$
\begin{aligned}
& p_{1}(\mathbf{x}, 0)=(2 \pi)^{-3 / 2} \int d^{3} k \frac{i \sqrt{\omega}}{\sqrt{2}}\left(a_{1}^{+}(\mathbf{k}) \mathrm{e}^{-i \mathbf{k x}}-a_{1}^{-}(\mathbf{k}) \mathrm{e}^{i \mathbf{k x}}\right), \\
& p_{2}(\mathbf{x}, 0)=-(2 \pi)^{-3 / 2} \int d^{3} k \frac{1}{2 \sqrt{\omega}}\left(a_{1}^{+}(\mathbf{k}) \mathrm{e}^{-i \mathbf{k x}}+a_{1}^{-}(\mathbf{k}) \mathrm{e}^{i \mathbf{k x}}\right), \\
& b(\mathbf{x}, 0)=(2 \pi)^{-3 / 2} \int d^{3} k \frac{1}{\sqrt{2 \omega}}\left(b^{+}(\mathbf{k}, 0) \mathrm{e}^{-i \mathbf{k x}}+b^{-}(\mathbf{k}) \mathrm{e}^{i \mathbf{k x}}\right), \\
& \dot{b}(\mathbf{x}, 0)=i(2 \pi)^{-3 / 2} \int d^{3} k \frac{\sqrt{\omega}}{\sqrt{2}}\left(b^{+}(\mathbf{k}) \mathrm{e}^{-i \mathbf{k x}}-b^{-}(\mathbf{k}) \mathrm{e}^{i \mathbf{k x}}\right) .
\end{aligned}
$$


(формулы для полей $e, \dot{e}$ получается из $(26)$ заменой $b, \dot{b} \rightarrow e, \dot{e}$ ) Введем следующие комбинации операторов $\hat{p}_{1,2}, \hat{\varphi}_{1,2}, \hat{b}, \hat{e}$, которые удовлетворяют перестановочным соотношениям операторов рождения и уничтожения:

$$
\begin{aligned}
\frac{\hat{p}_{2}(\mathbf{k}) \omega(\mathbf{k})+i \hat{p}_{1}(\mathbf{k})}{\sqrt{2 \omega}} & =-\hat{a}^{+}(\mathbf{k}), & & \frac{\hat{\varphi}_{1}(\mathbf{k}) \omega(\mathbf{k})+i \hat{\varphi}_{2}(\mathbf{k})}{\sqrt{2 \omega}}=\hat{\tilde{a}}^{-}(\mathbf{k}), \\
\frac{\hat{p}_{2}(\mathbf{k}) \omega(\mathbf{k})-i \hat{p}_{1}(\mathbf{k})}{\sqrt{2 \omega}} & =-\hat{a}^{-}(\mathbf{k}), & \frac{\hat{\varphi}_{1}(\mathbf{k}) \omega(\mathbf{k})-i \hat{\varphi}_{2}(\mathbf{k})}{\sqrt{2 \omega}} & =\hat{\tilde{a}}^{+}(\mathbf{k}), \\
\frac{\hat{b}(\mathbf{k}) \omega(\mathbf{k})+i \dot{\hat{b}}(\mathbf{k})}{\sqrt{2 \omega}} & =-\hat{b}^{+}(\mathbf{k}), & \frac{e(\mathbf{k}) \omega(\mathbf{k})+i \dot{\hat{e}}(\mathbf{k})}{\sqrt{2 \omega}} & =\hat{e}^{-}(\mathbf{k}), \\
\frac{\hat{b}(\mathbf{k}) \omega(\mathbf{k})-i \dot{\hat{b}}(\mathbf{k})}{\sqrt{2 \omega}} & =-\hat{b}^{-}(\mathbf{k}), & \frac{\hat{e}(\mathbf{k}) \omega(\mathbf{k})-i \hat{\hat{e}}(\mathbf{k})}{\sqrt{2 \omega}} & =\hat{e}^{+}(\mathbf{k}) .
\end{aligned}
$$

Заметим, что некоммутирующие пары образуют операторы $a, \hat{\tilde{a}}$ и $b, e$. Для осцилляторных переменных это определение совпадает с обычным.

В терминах введенных выше операторов асимптотический БРСТ-заряд можно записать в следующем виде:

$$
Q_{0}=i \int d^{3} k\left(\hat{a}^{+}(\mathbf{k}) \hat{b}^{-}(\mathbf{k})-\hat{b}^{+}(\mathbf{k}) \hat{a}^{-}(\mathbf{k})\right) .
$$

Стоит подчеркнуть, что состояния, порожденные операторами $\hat{\tilde{a}}$, не принадлежат фоковскому пространству. Однако это несущественно для физической интерпретации модели, так как часть $\widehat{\widetilde{H}}$ гамильтониана (20), которая зависит от переменных $\varphi_{1,2}, b, e$ и сопряженных моментов, БРСТ-точна: она может быть представлена в виде антикоммутатора БРСТ-заряда (24) с некоторым оператором $A$ :

$$
\widehat{\widetilde{H}}_{0}=\left[\widehat{Q}_{0}, \hat{A}\right]_{+}, \quad \hat{A}=\int d^{3} x\left(\hat{\varphi}_{2} \dot{\hat{e}}+\frac{\mu^{2}}{2} \hat{p}_{2} \hat{e}+\Delta \varphi_{1} \hat{e}\right) .
$$

Оператор $\widehat{\widetilde{H}}$ не дает вклада в вакуумные ожидания, вычисленные с помощью физических векторов, которые аннигилируются оператором $Q_{0}$ (аналогичная конструкция рассматривалась в работе [6]). Следовательно, он несуществен для энергии любого физического состояния, и мы можем определить вакуум как вектор, аннигилируемый операторами $\hat{a}^{-}, \hat{\tilde{a}}^{-}, \hat{b}^{-}, \hat{e}^{-}$.

Можно ввести оператор $\widehat{K}$ формулой

$$
\begin{aligned}
\widehat{K} & =\int d^{3} x\left(\hat{\varphi}_{1}(\mathbf{x}, 0) \dot{\hat{e}}(\mathbf{x}, 0)-\hat{\varphi}_{2}(\mathbf{x}, 0) \hat{e}(\mathbf{x}, 0)\right)= \\
& =-i \int d^{3} k\left(\tilde{\hat{a}}^{+}(\mathbf{k}) \hat{e}^{-}(\mathbf{k})-\hat{e}^{+}(\mathbf{k}) \tilde{\hat{a}}^{-}(\mathbf{k})\right) .
\end{aligned}
$$

Антикоммутатор операторов $\widehat{Q}_{0}$ и $\widehat{K}$ пропорционален числу нефизических мод, порожденных операторами $\hat{a}^{+}, \hat{\tilde{a}}^{+}, \hat{b}^{+}, \hat{e}^{+}$. Обычным образом можно показать, что любой вектор, удовлетворяющий условию (16), можно представить в виде

$$
|\psi\rangle_{\mathrm{phys}}=|\psi\rangle_{\mathrm{tr}}+Q_{0}|\chi\rangle
$$


где вектор $|\psi\rangle_{\text {tr }}$ содержит только возбуждения, отвечающие поперечным поляризациям поля Янга-Миллса. Матричный элемент любой наблюдаемой, вычисленный с помощью векторов $|\psi\rangle_{\text {phys }}$, совпадает с матричным элементом, вычисленным с помощью векторов $|\psi\rangle_{\mathrm{tr}}$.

Этим завершается доказательство того, что при $\alpha=0$ матричные элементы любой наблюдаемой, вычисленные с помощью лагранжиана (13) и физических состояний, удовлетворяющих условию (16), совпадают с матричными элементами в обычной теории Янга-Миллса. В то же время при $\alpha \neq 0$ данный лагранжиан описывает массивную калибровочно-инвариантную теорию, не имеющую инфракрасных сингулярностей.

\section{3. ОБСУЖДЕНИЕ}

Мы показали, что лагранжиан (9) можно однозначно проквантовать независимо от того, можно ли пользоваться теорией возмущений по константе связи. При любой величине константы связи он допускает однозначное каноническое квантование в калибровке $\phi^{a}=0$.

При $\alpha \neq 0$ этот лагранжиан не приводит к появлению инфракрасных сингулярностей и в то же время является калибровочно-инвариантным. Вводя какую-либо инвариантную ультрафиолетовую регуляризацию, например размерную или высшие ковариантные производные, мы получаем калибровочно-инвариантную регуляризацию, которая делает все величины конечными.

При $\alpha=0$ этот лагранжиан дает для матричных элементов любой наблюдаемой, вычисленных с помощью физических векторов, которые аннигилируются БРСТ-зарядом, те же значения, что и обычный лагранжиан Янга-Миллса. Разумеется, если вычисления выполняются в рамках теории возмущений, то при $\alpha=0$ вновь появляются инфракрасные сингулярности. Однако этот лагранжиан может служить отправной точкой для непертурбативных вычислений, в частности для вычислений, объясняющих явление невылета кварков.

Благодарности. Я хочу поблагодарить Андреа Квадри за чтение рукописи и полезные замечания. Эта работа была частично поддержана РФФИ (гранты № 11-01-00296_а и 11-01-12037 офи_м-2011), Программой поддержки ведущих научных школ и программой "Нелинейная динамика".

\section{Список литературы}

[1] А. А. Славнов, Л. Д. Фаддеев, ТМФ, 3:1 (1970), 18-23.

[2] А. А. Славнов, ТМФ, 161:2 (2009), 204-211.

[3] А. А. Славнов, ТМФ, 154:2 (2008), 213-219.

[4] A. A. Slavnov, JHEP, 08 (2008), 047, 11 pp., arXiv: 0807.1795.

[5] A. Quadri, A. A. Slavnov, JHEP, 07 (2010), 087, 22 pp., arXiv: 1002.2490.

[6] M. Henneaux, C. Teitelboim, Quantization of Gauge Systems, Princeton Univ. Press, Princeton, NJ, 1992. 